\title{
A familial case with brachydactyly type C with a GDF5 mutation
}

\author{
Hye Ryun Yeh¹, Beom Hee Lee, ${ }^{1,2}$ Ja Hye Kim¹, Ja Hyang Cho', Gu-Hwan Kim², Jae-Min Kim², In-Hee Choi², and Han-Wook Yoo ${ }^{1,2, *}$ \\ ${ }^{1}$ Department of Pediatrics, Asan Medical Center Children's Hospital, University of Ulsan College of Medicine, Seoul, Korea \\ ${ }^{2}$ Medical Genetics Center, Asan Medical Center, University of Ulsan College of Medicine, Seoul, Korea
}

\begin{abstract}
Brachydactyly type $\mathrm{C}$ is a limb malformation characterized by shortening of the second, third, and fifth middle and/or proximal phalanges, but it has variable phenotypic expressivity. Mutations in the growth differentiation factor-5 (GDF5) gene cause isolated brachydactyly $\mathrm{C}$. Herein, we report a familial case with isolated brachydactyly type $\mathrm{C}$ characterized by brachymesophalangy of both second and third digits, with a GDF5 missense mutation, and discuss the phenotypic variability of the condition. Identifying more cases with genetic confirmation will help elucidate the clinical and genetic characteristics of this condition in the Korean population.
\end{abstract}

Key words: Brachydactyly type C, GDF5, Mutation.

\section{Introduction}

Brachydactyly is caused by congenital skeletal disorders and characterized by the under development of or the absence of phalangeal and metacarpal bones. This condition can present as an isolated form or syndromic form combined with other congenital anomalies. Brachydactyly is mostly inherited in an autosomal dominant manner but with variable and overlapping phenotypic expressivity [1]. Brachydactyly is classified by phenotypic patterns into five brachydactyly types (A-E) that include several subtypes [2].

Among these, brachydactyly type $\mathrm{C}$ is characterized by shortening of the middle and/or proximal phalanges of the second, third, and fifth digits with a relatively normal fourth digit and can be associated with hypersegmentation of proximal or middle phalanges [3]. The causative gene of isolated bradydactyly type $\mathrm{C}$ is the growth differentiation factor-5 (GDF5) gene, which encodes a secreted growth factor involved in bone development and joint formation [1].

Isolated brachydactyly type $\mathrm{C}$ has been rarely reported in the Korean population, and only one case was confirmed via genetic testing. In this report, a familial case of a GDF5 mutation is described.

\section{Case}

A 54-year-old Korean man visited the genetics clinic because of bilateral finger anomalies. On both his hands, the second and third fingers were short, with ulnar deviation. The first, fourth, and fifth fingers were normal, and the fourth finger was the longest. The range of motion was normal in all the fingers. Otherwise, his upper and lower arms, lower extremities, and feet were normal. No facial dysmorphism was observed. Intellectual performance was normal. Neither of his parents were affected. He had two sons, one of whom had shortened the second and

Received: 29 May 2014, Accepted: 10 June 2014, Published: 30 June 2014

*Corresponding author: Han-Wook Yoo, M.D., Ph.D.

Department of Pediatrics, Asan Medical Center, University of Ulsan College of Medicine, 88 Olympic-ro 43-gil, Songpa-gu, Seoul 138-736, Korea.

Tel: +82-2-3010-3374, Fax: +82-2-473-3725, E-mail: hwyoo@amc.seoul.kr

Conflict of interest: We declare that we do not have any conflicts of interests.

(c) This is an open-access article distributed under the terms of the Creative Commons Attribution Non-Commercial License (http://creativecommons.org/licenses/by-nc/3.0/) which permits unrestricted non-commercial use, distribution, and reproduction in any medium, provided the original work is properly cited.

(c) Copyright 2014 by the Korean Society of Medical Genetics

www.e-kjgm.org 
third fingers. One relative of his father also had similar shortened digits.

We obtained radiographs of the hands of the 54-yearold patient and observed hypoplastic middle phalanges that both the second and third fingers showed ulnar deviation and hypersegemented third fingers on both the hands (Fig 1). The first, fourth, and fifth metacarpal and carpal bones of both the hands were normal. No obvious shortening of toes or metatarsals was observed. He had no other skeletal or nonskeletal dysmorphism.

Familial brachydactyly type C was suspected, and therefore, genetic testing for the GDF5gene was conducted using genomic DNA from the patient's peripheral leukocytes. Sequence analysis was performed for two exons and their intronic boundaries of the GDF5 gene using the BigDye terminator kit v3.1 (Applied Biosystems, Foster City, CA, USA). Sequence analyses were performed using an $\mathrm{ABI} 3130 x /$ genetic analyzer (Applied Biosystems), which revealed a missense mutation, c.1312C $>\mathrm{T}$, resulting in a change in the protein level of p.Arg438Cys that has previously been reported in isolated branchydactyly type C (Fig. 2) [4].

\section{Discussion}

Brachydactyly is a congenital malformation characterized by
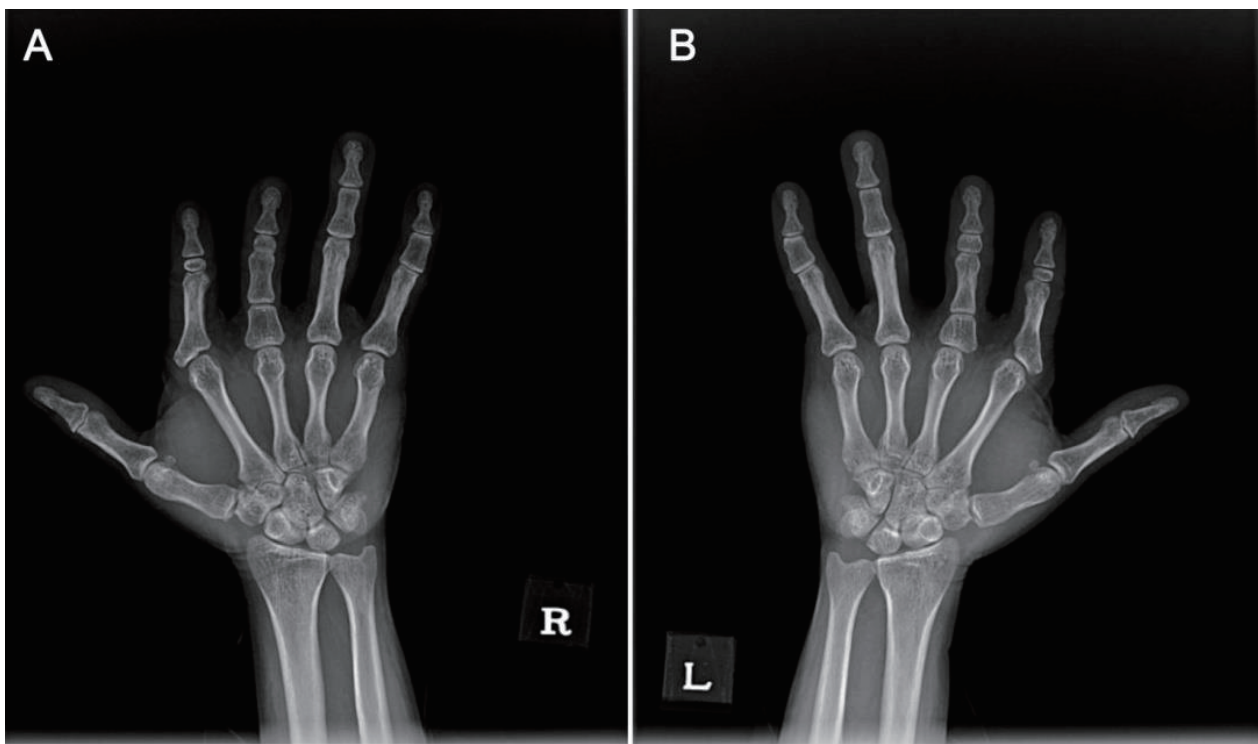

Fig. 1. Radiographs showed shortening of middle phalanges of the second and third digits as well as hypersegmentation of third digits. (A) Right hand, (B) left hand.

Partial seq. of GDF5 gene

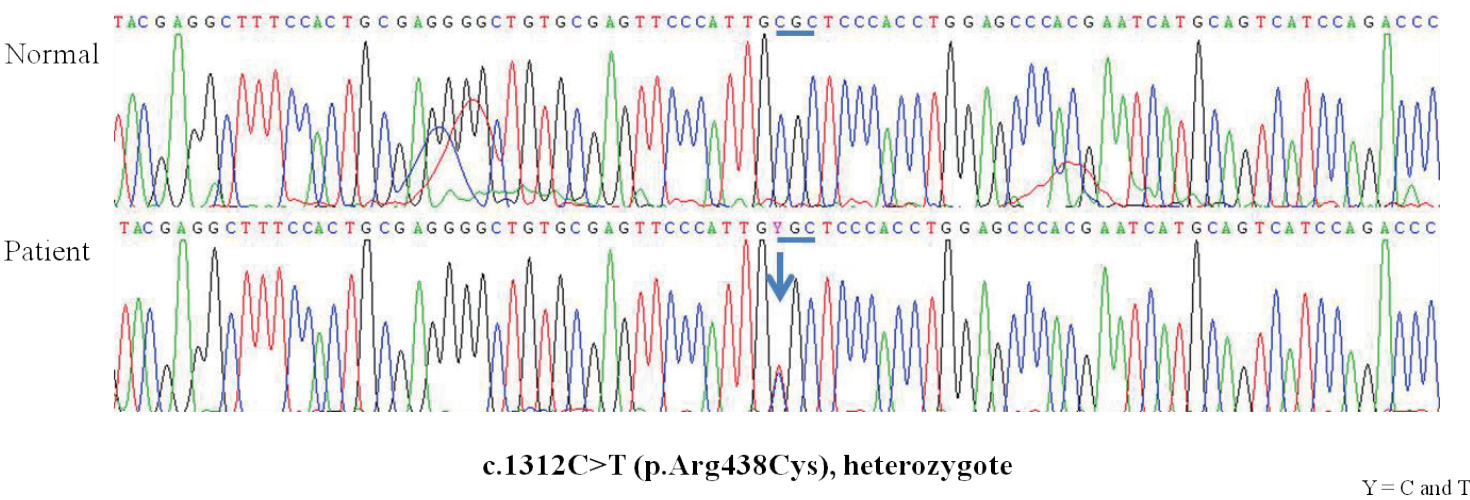

Fig. 2. Partial sequences of the GDF5 gene. The patient carries a heterozygote missense mutation, $c .1312 \mathrm{C}>\mathrm{T}$ ( $\mathrm{p}$. Arg $438 \mathrm{Cys}$ ). The upper electrogram is of the normal control and the lower is of the patient. The mutation site is indicated (arrow). Two nucleotides, "C" and "T," are presented as "Y." 
shortening of digits due to underdevelopment of the tubular bones in the hands and/or feet. This condition mainly presents as an isolated autosomal dominant anomaly, but may also present as a combination of features associated with syndromic disorders. Brachydactyly has been classified into five main types (A-E) and their subtypes, based on the observed anatomy.

Brachydactyly type $\mathrm{C}$ is characterized by shortening of the middle phalanges of the second, third, and fifth fingers or the first metacarpal bone and other accompanying hand abnormalities, such as ulnar deviation of the index finger and hypersegmentation of proximal or middle phalanges [5]. This type of brachydatyly is rarer than others, and no epidemiologic data have been reported for the Korean population. So far, other skeletal features, such as polydactyly, Madelung deformity, talipes valgus or equinovarus, shortening or absence of the middle phalanges of the toes, hip dysplasia, and short stature have also been observed in individuals with brachydactyly type C [5]. However, a wide range of clinical and radiological variability has been reported in cases confirmed on the basis of the same GDF5 gene mutation resulting in the presentation of brachydactyly type C [3]. As for other brachydactyly types, deformity is confined to shortening of middle phalanges in type $A$, whereas the distal phalanges are mainly affected in type $B$ [2]. Type $D$ is characterized by shortening of the distal phalanges of the thumb, and shortening of the metacarpals are variable in type E[2].

Our patient had brachymesophalangy of both the second and third digits with hypersegementation of the third digit and no additional hand deformities, including shortening of the fifth finger and the first metacarpal bone or hyperphalangy, which are consistent with the characteristics of brachydactyly type $\mathrm{C}$.

GDF5 is a protein that plays an important role in limb development, including bone and joint formation [6]. The GDF5 gene is located on 20q11.2 and consists of two exons encoding 501 amino acids. This gene consists of a signal peptide, the prodomain, and the active signaling domain. GDF5, a member of the TGFß superfamily, regulates cell growth and differentiation by posttranslational modification, which causes dimer formation and regulation of target gene expression [6].

Until now, more than 20 different GDF5 mutations have been found to be associated with brachydactyly (www.hgmd. cf.ac.uk). Most mutations in the prodomain are frameshift mutations that mainly result in brachydactyly type $C$ [4]. On the other hand, mutations in the active domain, most of which are missense mutations, have been identified in brachydactyly type A1, brachydactyly type A2, Du Pan syndrome, Grebe type chondrodysplasia, Hunter-Thompson syndrome, and multiple synostoses syndrome 2, in addition to brachydactyly type C [4].

The mutation identified in our patient, c.1312C>T (p.Arg438Cys), is located in the active signaling domain. This mutation has been previously described, but the precise mechanism by which this mutation alters the function of the active domain remains unknown [4]. Recent studies suggested that mutations in the GDF5 gene lead to loss of function or reduce GDF5 protein levels below the biological threshold required for normal digit development [4]. Additionally, environmental or epigenetic factors can also effect digit development and associated phenotypic variability [5]. Although brachydactyly type $\mathrm{C}$ is inherited in an autosomal dominant manner, it is difficult to predict the phenotypic severity of an affected fetus by prenatal genetic counseling because of its phenotypic variability [7]. This phenotypic variability was also observed in our patient's family members; although other family members were not genetically tested, another relative and his son had phenotypic abnormalities but the patient's father did not.

The first Korean case with brachydactyly type $\mathrm{C}$ confirmed by genetic testing had the same mutation as our patient. This con was sporadic, and the patient had short middle phalanges of the second, third, and fifth digits. To the best of our knowledge, the case reported here is the second Korean case with the same mutation, c.1312C>T (p.Arg438Cys) [3]. Identification of more cases with brachydactyly type $C$ will help improve our understanding of the clinical characteristics and mutation spectrum of this disorder in the Korean population.

\section{Acknowledgements}

We thank our patient and his family members for their participation in this study. This research was supported by a grant from the National Research Foundation of Korea, funded by the Ministry of Education, Science and Technology (Grant No. 20110019674).

\section{References}

1. Holder-Espinasse M, Escande F, Mayrargue E, Dieux-Coeslier A, Fron D, Doual-Bisser A, et al. Angel shaped phalangeal dysplasia, hip dysplasia, and positional teeth abnormalities are part of the brachydactyly $C$ spectrum associated with CDMP-1 mutations. J Med Genet 2004;41:e78.

2. Girirajan S, Elsea SH. Brachydactyly A1: new relatives for old families? J 
Genet 2005:84:95-8.

3. Seo SH, Park MJ, Kim SH, Kim OH, Park S, Cho SI, et al. Identification of a GDF5 mutation in a Korean patient with brachydactyly type $\mathrm{C}$ without foot involvement. Ann Lab Med 2013;33:150-2.

4. Everman DB, Bartels CF, Yang Y, Yanamandra N, Goodman FR, MendozaLondono JR, et al. The mutational spectrum of brachydactyly type C. Am J Med Genet 2002;112:291-6.

5. Gutiérrez-Amavizca BE, Brambila-Tapia $\mathrm{AJ}$, Juárez-Vázquez $\mathrm{Cl}$, Holder-
Espinasse M, Manouvrier-Hanu S, Escande $F$, et al. A novel mutation in CDMP1 causes brachydactyly type $C$ with "angel-shaped phalanx". A genotype-phenotype correlation in the mutational spectrum. Eur J Med Genet 2012;55:611-4.

6. Yang W, Cao L, Liu W, Jiang L, Sun M, Zhang D, et al. Novel point mutations in GDF5 associated with two distinct limb malformations in Chinese: brachydactyly type C and proximal symphalangism. J Hum Genet 2008;53:368-74.

7. TemtamySA, Aglan MS. Brachydactyly. Orphanet J Rare Dis 2008;3:15. 